\title{
Inadvertent hypothermia during the perioperative period
}

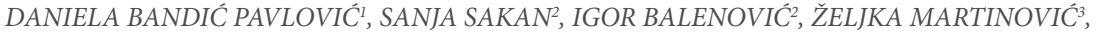 \\ ROBERT BARONICA ${ }^{2}$, DINKO TONKOVIĆ ${ }^{1}$, MLADEN PERIĆ ${ }^{1}$ \\ ${ }^{1}$ School of Medicine, University of Zagreb \\ ${ }^{2}$ University Hospital Centre Zagreb \\ ${ }^{3}$ Ministry of Labour and Pension System; Medical Assessors Department
}

Corresponding author:

Daniela Bandić Pavlović

School of Medicine, University of Zagreb, Croatia

Phone: +38598515058

E-mail: daniela.bandic.pavlovic@mef.hr

\section{ABSTRACT}

Inadvertent hypothermia, which is defined as temperature below $36^{\circ} \mathrm{C}$, is common in the perioperative setting. Patients under general or regional anaesthesia have impaired temperature regulation/homeostasis. Temperature monitoring should be an established standard for all procedures that last more than 30 minutes. Unfortunately, study shows that it is not a common practice in European hospitals. Passive and active patient warming should be used to prevent and treat hypothermia. Warming should start in the preoperative period and last throughout all perioperative phases. In that way, well-known complication of hypothermia should be prevented. Cardiac event, coagulopathy and wound infection are the leading causes of delayed discharge and more adverse outcomes related to hypothermia. It is especially important to undertake all necessary intervention procedures to prevent hypothermia in a group of patients with known high number of risk factors for hypothermia. Ambient temperature, an important risk factor, should be monitored and maintained at about $21^{\circ} \mathrm{C}$. According to reviewed evidence, the protocol to prevent, monitor and treat hypothermia should be established. Further studies about the implementation of temperature monitoring and regulation are needed in order to raise awareness about this issue.

Key words: hypothermia, core temperature, anaesthesia, warming devices, prevention, treatment

\section{INTRODUCTION}

Temperature homeostasis is regulated by the thermoregulatory system, which maintains normothermia. Impaired normothermia activates a defensive autonomic response. Hypothermia leads to activation of vasoconstriction at the beginning followed by shivering, while hyperthermia leads to sweating and vasodilatation at the same time. (1) However, anaesthesia impairs that regulatory mechanism. (2 4) Inadvertent hypothermia is common in the perioperative setting. According to some authors, the prevalence is 50 to $90 \%$. (5) Temperature monitoring is necessary not only to avoid hypothermia but to avoid hyperthermia as well, along with the resulting morbidities. Different warming techniques have been developed. Nonetheless, the incidence of hypothermia after surgery is still high. The major problem with regard to hypothermia has not to do with monitoring or treatment devices, but rather with the awareness of the importance of temperature monitoring and its implementation in standard practice. The aim of this article is to provide answers on the main questions associated with hypothermia.

\section{DISCUSSION}

\section{Why treat hypothermia?}

Maintaining core temperature in the perioperative setting is of the utmost importance for the course of the disease. Hypothermia, which is defined as core temperature below $36^{\circ} \mathrm{C}$, has impact on several systems. The prevalence of cardiac incidents, myocardial ischemia and tachycardia is increased. Development of coagulopathy with greater blood loss even for $0.5^{\circ} \mathrm{C}$ occurs as a result of platelet and clotting factor dysfunction. Impaired immune response increases the risk of wound infections. Consequently, wound healing is delayed. Furthermore, waking up from anaesthesia is delayed owing to the change in the pharmacokinetics of anaesthetic drugs, especially muscle relaxants. Overall, this will result in delayed discharge and a longer hospitalization period. Ultimately, normothermia improves patient outcomes. $(2,6)$

What are the risk factors?

Several studies defined risk factors for hypothermia. Factors that cannot be influenced are patient characteristics such as advanced age, sex, low body weight, existing diabetic neuropathia. Others can be changed, i.e. preoperative body temperature. Studies confirmed the following intraoperative risk factors: emergency surgery, major surgery with large open surface, duration of surgery or anaesthesia for more than two hours, epidural anaesthesia combined with general anaesthesia, and over four litres of applied intravenous fluid. Additionally, low ambient temperature, a well-recognized risk factor, should be monitored and maintained at least at $21^{\circ} \mathrm{C}$, which is comfortable enough for work and without increased risk of infection. When active warming has started, ambient temperature can be decreased for the staff. (7)

\section{What is the reaction to hypothermia?}

The first reaction to hypothermia is vasoconstriction in the arteriovenous shunt, primarily located in fingers and toes. The purpose is to redirect blood flow towards those organs that are centrally mediated and to preserve the temperature of the core. Shivering is secondary, which is 
involuntary muscle contraction that increases metabolic rate, and thus increases body temperature, but ultimately without a significant increase in core temperature in contrast to muscle activity in exercise. Shivering of thermogenic origin should be differentiated from tremor related with other causes such as volatile anaesthetics, pain or inhibitors of dopamine receptors. $(3,8)$

\section{Why are patients in anaesthesia prone to hypothermia?}

Both general and regional anaesthesia make a patient prone to hypothermia. General anaesthetics used for induction disturb the heat balance by decreasing the vasoconstriction threshold. Thus, arteriovenous shunts are still open despite the lower core temperature. Normal defence response is present, but it is activated too late. Therefore, the rate of heat loss is greater so it leads to hypothermia. In anaesthesia the metabolic heat production is also decreased. Low ambient temperature additionally worsens hypothermia, more with radiation and convection of heat and less with evaporation and conductive loss from patients to environment. Net effect is hypothermia which further decreases the metabolic heat rate and activates a 'circulus vitiosus'. In one moment, the vasoconstriction threshold temperature is reached, vasoconstriction is activated, core temperature is hypothermic, but has now reached a steady state. $(2,4,8,9)$

Similar situation is visible in the central regional block with spinal or epidural anaesthesia. Thermal homeostasis is disturbed through central and peripheral mechanisms. The mechanism of hypothermia is related to sympathetic blockade resulting in vasodilatation and heat loss. On the other hand, vasoconstriction and shivering as defence mechanisms are present in upper body. The mechanism of centrally mediated hypothermia is not clear, but studies show that temperature threshold of vasoconstriction and shivering is decreased. In contrast to general anaesthesia, a steady state of core temperature cannot be reached owing to blocked vasoconstriction in the lower part of the body. $(10-12)$

\section{Where and when to monitor core tem- perature?}

General recommendation stresses the importance of intraoperative temperature monitoring. Core temperature should be measured in highly perfused tissue, i.e. na- sopharynx, tympanic membrane, pulmonary artery or distal oesophagus. (9) After 40 minutes of anaesthesia, core temperature decreases by $1^{\circ} \mathrm{C}$. Therefore, all procedures that last longer than 30 minutes should include temperature monitoring. (6)

\section{What kind of warming treatments are mostly used?}

There are two main treatment options for managing hypothermia, the passive or the active approach. Surgical draping, cotton blankets and metallized plastic covers are representative of the passive approach. Forced-air warming devices, resistive heating blankets and circulating water mattresses are some of the available gear in the active approach. (6) Moola investigated the most effective system among different passive and active devices, any type of linen or cover, aluminium foil wraps, forced-air warming devices, radiant warming devices and fluid warming devices. (5) In conclusion, forced air warming proved to be the most effective. (5)

Several studies emphasize that hypothermia should be prevented. $(6,7,13,14)$ Therefore, patient pre-warming should start in the preoperative period and last throughout the perioperative period. The preoperative period is defined as 1 hour before induction of anaesthesia. This period also covers the transport from the ward to the operating room. In this period patient's legs and arms should be warmed for them to feel comfortable. Active warming in the preoperative phase is started if the temperature is lower than $36^{\circ} \mathrm{C}$. Otherwise is not necessary until operating room. $(13,14)$ Mostly a combined active warming system is used in the operating room. Forced air warming devices and intravenous fluid warming system at room temperature successfully prevent and treat hypothermia. (5, 15) However, the intravenous fluid warming system just prevents hypothermia due to the fact that four litres of saline or four blood units will decrease the temperature by $1^{\circ} \mathrm{C}$. (16)

Warttig et al. reviewed randomised controlled trials of postoperative warming. They concluded that forced air warming is efficient in the postoperative period, but there is a lack of evidence both on other benefits and the possible side effects as well. (17) Furthermore, other rewarming systems are not investigated sufficiently. (17)

\section{Our reality:}

In our hospital, temperature monitors are not the standard equipment for anaesthesia patients who undergo procedures longer than 30 minutes. A European survey from 2004 also showed deflating results. Temperature monitoring was used in every fifth patient, significantly more frequently in general anaesthesia when compared to regional anesthesia. (13) Perioperative patient warming is not carried out at all. It starts in the operating room, with the thermometer placed in the hypopharynx to measure core temperature. Economic conditions also influence medical criteria. Active warming devices are expensive. Patients with major abdominal and vascular procedures, patients with polytrauma and critically ill patients are recognized as especially vulnerable groups prone to hypothermia. Therefore, temperature monitoring and active warming is part of their routine care. It is common practice to use a temperature management blanket for adults (positioned under the body) and a fluid warming device for intravenous fluid and blood warming. Our practice is also to maintain ordinary ambient temperature at around $21^{\circ} \mathrm{C}, 22^{\circ} \mathrm{C}$.

\section{CONCLUSION}

Hypothermia in the perioperative setting is the result of exposure to low ambient temperature in the operating room and of anaesthesia, which impairs the thermoregulatory defence mechanism. In general anaesthesia the mechanism is activated with delay, and in regional anaesthesia this mechanism is effective only in the nonblocked body part. $(2,9,12)$ Unfortunately, hypothermia is a neglected problem that is present in a large number of surgically treated patients. $(5,13)$ The solution is to raise awareness of the process of hypothermia and its complications during surgical procedures. Trials stress the importance of pre-warming, which means taking care of body temperature one hour before induction of anaesthesia. $(2,5,17)$ According to recommendations, core temperature monitoring and treatment should start in the preoperative period for surgeries that last more than half an hour. $(6,14)$ Eventually, temperature monitoring and active patient warming should become part of standard basic care for patients, such as measuring arterial pressure or electrocardiography. According to reviewed evidence, the protocol to prevent, monitor and treat hypothermia should be established. 


\section{REFERENCES}

1. Lenhardt R. The Effect of anesthesia on body temperature control. Frontiers in Bioscience. 2010;S2:1145-54.

2. Horosz B, Malec-Milewska M. Inadvertent intraoperative hypothermia. Anaesthesiology Intensive Therapy. 2013;45(1):38-43.

3. Hales JRS. Skin arteriovenous anastomoses, their control and role in thermoregulation. In. Cardiovascular Shunts: Phylogenetic, Ontogenetic and Clinical Aspects. Eds. K Johansen, W Burggren. Munksgaard, Copenhagen, 1985:433-451.

4. Matsukawa T, DI Sessler, AM Sessler, M Schroeder, M Ozaki, A Kurz, C Cheng. Heat flow and distribution during induction of general anesthesia. Anesthesiology.1995;82, 662-73.

5. Moola S, Lockwood C. Effectiveness of strategies for the management and/or prevention of hypothermia within the adult perioperative environment. Int J Evid Based Healthc. 2011;9(4):337-45.

6. Putzu M, Casati A, Berti M, Pagliarini G, Fanelli. Clinical complications, monitoring and management of perioperative mild hypothermia: anesthesiological features. Acta Biomed. 2007;78(3):163-9.

7. Kongsayreepong S, Chaibundit C, Chadpaibool J, Komoltri C, Suraseranivongse S, Suwannanonda P, Raksamanee EO, Noocharoen P, Silapadech A, Parakkamodom S, Pum-In C, Sojeoyya L. Predictor of core hypothermia and the surgical intensive care unit. Anesth Analg. 2003;96(3):826-33.

8. Díaz M, Becker DE. Thermoregulation: Physiological and Clinical Considerations during Sedation and General Anesthesia. Anesth Prog. 2010; 57(1): 25-33.

9. Insler SR, Sessler DI. Perioperative thermoregulation and temperature monitoring. Anesthesiol Clin. 2006;24(4):823-37.

10. Leslie K, DI Sessler. Reduction in the shivering threshold is proportional to spinal block height. Anesthesiology. 1996; 84: 1327-31.

11. Ozaki M, A Kurz, DI Sessler, R Lenhardt, M Schroeder, A Moayeri, KM Noyes, E Rotheneder. Thermoregulatory thresholds during spinal and epidural anesthesia. Anesthesiology.1994;81:282-8.

12. Kurz A, DI Sessler, M Schroeder, M Kurz: Thermoregulatory response thresholds during spinal anesthesia. Anesth Analg.1993;77: 721-6.

13. The thermoregulation in Europe, Monitoring and Managing Patient Temperature (TEMMP) study group. Survey on intraoperative temperature management in Europe. EurJ Anaesthesiol 2007.

14. NICE. Inadvertent perioperative hypothermia: The management of inadvertent perioperative hypothermia in adults. London: National Institute for Health and Clinical Excellence Guideline 65, 2008.

15. Brauer A, M Quintel. Forced-air warming: technology, physical background and practical aspects. Curr Opin Anaesthesiol. 2009;22:769-74.

16. Sessler DI. Consequences and treatment of perioperative hypothermia. Anesth Clin North Am 1994; 12: 425-56.

17. Warttig S, Alderson P, Campbell G, Smith AF. Interventions for treating inadvertent postoperative hypothermia. Cochrane Database Syst Rev. 2014: 20;11 